\title{
Estimation of Hazard Rate and Mean Residual Life Ordering for Fuzzy Random Variable
}

\author{
S. Ramasubramanian and P. Mahendran \\ Department of Mathematics, Anna University, BIT Campus, Tiruchirappalli 620024, India \\ Correspondence should be addressed to P. Mahendran; p.mahendran@mail.aubit.edu.in
}

Received 4 March 2015; Accepted 23 June 2015

Academic Editor: Józef Banaś

Copyright (C) 2015 S. Ramasubramanian and P. Mahendran. This is an open access article distributed under the Creative Commons Attribution License, which permits unrestricted use, distribution, and reproduction in any medium, provided the original work is properly cited.

\begin{abstract}
$L_{2}$-metric is used to find the distance between triangular fuzzy numbers. The mean and variance of a fuzzy random variable are also determined by this concept. The hazard rate is estimated and its relationship with mean residual life ordering of fuzzy random variable is investigated. Additionally, we have focused on deriving bivariate characterization of hazard rate ordering which explicitly involves pairwise interchange of two fuzzy random variables $X$ and $Y$.
\end{abstract}

\section{Introduction}

Fuzzy random variables generalize random variables and random sets. Kwakernaak [1] introduced the concept of a fuzzy random variable as a function $X: \Omega \rightarrow F(R)$ where $(\Omega, A, P)$ is a probability triple and $F(R)$ denotes the set of all canonical fuzzy numbers. Puri and Ralescu [2] defined the notion of fuzzy random variable as a function $X: \Omega \rightarrow F\left(R^{n}\right)$ where $(\Omega, A, P)$ is a probability space and $F\left(R^{n}\right)$ indicates all functions $U: R^{n} \rightarrow[0,1]$. In real life situations, life times of machines and components are expressed in terms of linguistic variables with imprecise boundaries. In such cases, fuzzy random variables are only capable of dealing with the vague data. In survival or reliability studies, the hazard rate is an important characteristic. In life testing situations, the expected additional life time given that a component has survived until $t$ is function of $t$ known as mean residual life. The hazard rate and mean residual life have been employed in life length studies by various authors. The properties of mean residual function can be found in Ruiz and Navarro [3], Gupta [4], Gupta and Olcay Akman [5], and Finkelstein [6]. Akbari et al. [7] described a bootstrap method for estimating the variance that was designed for the testing of hypotheses problem for fuzzy data based on the $L_{2}$ metric.

The concept of aging plays a significant role in reliability and maintenance theory in demography and in other life sciences. Notions of aging describe how an object improves or deteriorates with the passage of time. The concept of fuzzy reliability is increasingly receiving expanded interpretation in the literature. Hence, this paper deals with the fuzzy hazard rate, bivariate functional representation of hazard rate ordering of fuzzy random variables, fuzzy mean residual life functions, and their corresponding stochastic orderings for their meaningful and realistic endeavors. It is established that increasing failure rates in the fuzzy sense imply the decrease in fuzzy mean residual lives.

Shanthikumar and Yao [8] have introduced the concept of bivariate characterization of stochastic order relation with an application to single machine stochastic problem for random variables. Imprecise processing times, different work places, environmental conditions, and so forth, which are the factors involved in job scheduling problem, are fuzzy in nature. Itoh and Ishii [9] have proposed one machine scheduling problem with fuzzy random environment. Piriyakumar and Ramasubramanian [10] discussed bivariate characterization of likelihood ratio ordering of fuzzy random variables. Hence, we have extended our work to bivariate characterization of hazard rate ordering of fuzzy random variables.

This paper is organized in the following manner. The preliminaries have been explained in Section 2. In Section 3, triangular fuzzy number is defined; the mean and variance of a fuzzy random variable have been obtained by using 
$L_{2}$ metric. In Section 4, the fuzzy hazard rate function is determined. Section 5 focuses on the concept of fuzzy hazard rate function and fuzzy mean residual life function and their relationship. Bivariate characterization of hazard rate ordering which explicitly involves the interchange of fuzzy random variables is proposed in Section 6.

\section{Preleminaries}

Definition 1. Let $X$ be a universal set; then a fuzzy set $\widetilde{A}=$ $\left\{\left(x, \mu_{A}(x)\right) / x \in X\right\}$ of $X$ is defined by its membership function $\mu_{\widetilde{A}}: X \rightarrow[0,1]$.

Definition 2. For each $0 \leq \alpha \leq 1$, the $\alpha$-cut set of $\widetilde{A}$ is denoted by $\widetilde{A}_{\alpha}=\left\{x \in X: \mu_{\widetilde{A}}(x) \geq \alpha\right\}$.

Definition 3. A fuzzy number is a fuzzy set of $R$ such that the following conditions are satisfied:

(i) $\widetilde{A}$ is normal if there exists $x \in X$ such that $\mu_{\widetilde{A}}(x)=1$.

(ii) $\widetilde{A}$ is called convex if $\mu_{\widetilde{A}}\left(\lambda x_{1}+\left(1-\lambda x_{2}\right)\right) \geq$ $\min \left(\mu_{\widetilde{A}}\left(x_{1}\right), \mu_{\widetilde{A}}\left(x_{2}\right)\right)$.

(iii) $\widetilde{A}$ is called upper semicontinuous with compact support; that is, for every $\epsilon>0$, there exists $\delta>0$; $|x-y|<\delta \Rightarrow \mu_{\widetilde{A}}(x)<\mu_{\widetilde{A}}(y)+\epsilon$.

(iv) The $\alpha$-cut of a fuzzy number is a closed interval denoted by $A_{\alpha}=\left[A_{\alpha}^{L}, A_{\alpha}^{U}\right]$, where $A_{\alpha}^{L}=\inf \{x \in$ $\left.R ; \mu_{\widetilde{A}}(x) \geq \alpha\right\}$ and $A_{\alpha}^{U}=\sup \left\{x \in R ; \mu_{\widetilde{A}}(x) \geq \alpha\right\}$.

(v) If $\widetilde{A}$ is a closed and bounded fuzzy number with $A_{\alpha}^{L}$, $A_{\alpha}^{U}$ and its membership function is strictly increasing on $\left[A_{\alpha}^{L}, A_{1}^{L}\right]$ and strictly decreasing on $\left[A_{1}^{U}, A_{\alpha}^{U}\right]$, then $\widetilde{A}$ is called canonical fuzzy number.

Given a real number $x$, we can induce a fuzzy number $\tilde{x}$ with the membership function $\mu_{\tilde{x}}(r)$ such that $\mu_{\tilde{x}}(x)=1$ and $\mu_{\tilde{x}}(r)<1$ for $r \neq x$. We say $\tilde{x}$ is a fuzzy real number induced by the real number $x$. Let $F(\mathfrak{R})$ be a set of all fuzzy numbers induced by the real number system $R$. We define the relation $\sim$ on $\mathscr{F}_{R}$ as $\tilde{x}^{1} \sim \tilde{x}^{2}$ if and only if $\tilde{x}^{1}$ and $\tilde{x}^{2}$ are induced by the same real number $\tilde{x}$. Then $\sim$ is an equivalence relation. This induces the equivalence classes $[\tilde{x}]=\{\tilde{a} / \widetilde{a} \sim \tilde{x}\}$. The set of all equivalence classes induced by $\sim$ is denoted by $F(\mathfrak{R}) / \sim$.

Then the cardinality of $(F(\Re) / \sim)$ is equal to the cardinality of the real number system $R$. This claim is justified by the bijection map $R \rightarrow(F(\Re) / \sim)$ specified by $x \rightarrow[\tilde{x}]$. We call $(F(\Re) / \sim)$ the fuzzy real number system. For practical reasons, we consider any one of the elements from the equivalence class $[\tilde{x}]$ to form the fuzzy real number system $(F(\Re) / \sim)$. That is, $(F(\Re) / \sim)=\{\widetilde{A}: \widetilde{A} \in[\widetilde{A}], \widetilde{A}$ is the only element from $[\widetilde{A}]\}$.

If the fuzzy real number system $(F(\Re) / \sim)$ consists of canonical fuzzy real numbers, then we call $(F(\Re) / \sim)$ the canonical fuzzy real number system.

Definition 4. We define the distance between two canonical fuzzy numbers using $L_{2}$ metric in the fuzzy real number system $(F(\mathfrak{R}) / \sim)$, where $\sim$ is the equivalence relation inducing equivalence classes $(F(\Re) / \sim)=\{\widetilde{A}: \widetilde{A} \in[\widetilde{A}], \widetilde{A}$ is the only element from $[\widetilde{A}]\}$.

For each $\alpha$-cut of $\widetilde{A} \in F\left(\Re^{n}\right)$, the support function $S_{\widetilde{A}_{\alpha}}$ is defined as $S_{\widetilde{A}_{\alpha}}(t)=\operatorname{Sup}_{a \in \widetilde{A}_{\alpha}}\langle\langle a, t\rangle\rangle, t \in S^{n-1}$ where $S^{n-1}$ the $(n-1)$ dimensional unit sphere in $\mathfrak{R}^{n}$. Using support function, we define $L_{2}$ metric $\delta_{2}(\widetilde{A}, \widetilde{B})=\left(n \int_{0}^{1}\left(\rho_{2}\left(\widetilde{A}_{\alpha}, \widetilde{B}_{\alpha}\right)\right)^{2}\right)^{1 / 2}$, where $\widetilde{A}, \widetilde{B} \in F\left(\Re^{n}\right)$ and $\rho_{2}\left(\widetilde{A}_{\alpha}, \widetilde{B}_{\alpha}\right)=\left(\int_{S^{n-1}}\left|S_{\widetilde{A}_{\alpha}}(t)-S_{\widetilde{B}_{\alpha}}(t)\right|^{2} \mu(d t)\right)^{\mathbf{1 / 2}}$. Here $\mu$ denotes the Lebesgue measure on $S^{n-1}$ [7].

\section{Mean and Variance of Fuzzy Random Variables}

In real life situations, the life time of consumer based products is expressed by the masses in terms of linguistic variables rather than specific numeric quantities. Such opinions of the masses are not only linguistic but also intermixed with vagueness. This vagueness arises due to the dimness of perception. In such a situation, imprecise data are called into play. In such cases, fuzzy random variables are only capable of dealing with the vague data.

Define the left and right shape functions $L$ and $R$ as $L$ : $R^{\geq 0} \rightarrow[0,1]$ and $R: R^{\geq 0} \rightarrow[0,1]$ with $L(0)=R(0)=1$, that is, a fuzzy set $\tilde{x}$ with

$$
\mu_{\tilde{x}}(x)= \begin{cases}L\left(\frac{a-x}{l}\right), & x<a, \\ R\left(\frac{x-a}{r}\right), & x>a, \\ 1, & x=a .\end{cases}
$$

Definition 5. Let $(F(\Re) / \sim)$ be a fuzzy real number system and let $\widetilde{X}: \Omega \rightarrow(F(\Re) / \sim)$ be a closed fuzzy valued function. $\widetilde{X}$ is a fuzzy random variable if and only if $\widetilde{X}_{\alpha}^{L}$ and $\widetilde{X}_{\alpha}^{U}$ are random variables of $\alpha$ [2].

Definition 6. If $\widetilde{X}$ is a fuzzy random variable, the expected value of $\widetilde{X}$ is the unique fuzzy subset of $\Re$ denoted by $\widetilde{E}(X)$ such that, for all $\alpha \in[0,1]$, one has $E_{\alpha}(\widetilde{X})=E\left(\widetilde{X}_{\alpha}\right)=$ $\left[E\left[X_{\alpha}^{L}\right], E\left[X_{\alpha}^{U}\right]\right]$.

Definition 7. The variance of a fuzzy random variable $\widetilde{X}$ on the basis of $L_{2}$ metric is defined as $V(\widetilde{X})=E\left[\delta_{2}^{2}(\widetilde{X}\right.$, $\widetilde{E}(\widetilde{X})]$, where $\delta_{2}^{2}\left(\widetilde{x_{1}}, \widetilde{x_{2}}\right)=\left(a_{1}-a_{2}\right)^{2}+(1 / 2) \int_{0}^{1}\left(L^{-1}(\alpha)\right)^{2} d \alpha$ $\left(l_{1}-l_{2}\right)^{2}+(1 / 2) \int_{0}^{1}\left(R^{-1}(\alpha)\right)^{2} d \alpha\left(r_{1}-r_{2}\right)^{2}-\int_{0}^{1} L^{-1}(\alpha) d \alpha\left(a_{1}-\right.$ $\left.a_{2}\right)\left(l_{1}-l_{2}\right)+\int_{0}^{1} R^{-1}(\alpha) d \alpha\left(a_{1}-a_{2}\right)\left(r_{1}-r_{2}\right)$ [7].

\section{Hazard Rate Function}

Hazard rate is the frequency with which a component fails. The failure rate of a system usually depends on time with the rate varying over the life cycle of the system.

Let $\widetilde{X_{1}}, \widetilde{X_{2}}, \ldots, \widetilde{X_{n}}$, be independent and identically distributed fuzzy random variables.

As $\tilde{\mu}=E\left(\widetilde{X_{n}}\right)$ and $S_{n}^{2} \rightarrow \operatorname{Var}(\widetilde{X})$ by central limit theorem, $\widetilde{X} \sim(\bar{X}-\mu) /\left(S_{n} / \sqrt{n}\right)$ follows normal distribution 
$N\left(\widetilde{\mu}, S_{n} / \sqrt{n}\right)$ as $n \rightarrow \infty$. Generally, the random variable $T$ is said to be normally distributed with mean $\mu$ and variance $\sigma^{2}$ when the probability density of $T$ is $f(t)=$ $(1 / \sigma \sqrt{2 \pi}) e^{-(t-\mu)^{2} / 2 \sigma^{2}}$.

The $N(0,1)$ distribution is called the standard normal distribution. The distribution function of the standard normal distribution is usually denoted by $\varphi(\cdot)$. The probability density of the standard normal distribution is $\varphi(t)=$ $(1 / \sqrt{2 \pi}) e^{-t^{2} / 2}[11]$.

The distribution function may be written as $F(t)=$ $\operatorname{Pr}(T \leq t)=\varphi((t-\mu) / \sigma)$.

The reliability function is $R(t)=1-\varphi((t-\mu) / \sigma)$.

Then the failure rate function of the normal distribution is

$$
\lambda(t)=-\frac{R^{\prime}(t)}{R(t)}=\frac{1}{\sigma} \cdot \frac{\varphi(t-\mu / \sigma)}{1-\varphi((t-\mu) / \sigma)} .
$$

Using this concept, we can determine the failure rate function of the normal distribution with mean $\widetilde{\mu}$ and variance $S_{n} / \sqrt{n}$ as

$$
\lambda(t)=\frac{1}{\left(S_{n} / \sqrt{n}\right)} \cdot \frac{\varphi\left((t-\tilde{\mu}) /\left(S_{n} / \sqrt{n}\right)\right)}{1-\varphi\left((t-\tilde{\mu}) /\left(S_{n} / \sqrt{n}\right)\right)},
$$

where the mean and variance have already been determined in the previous Section 3.

\section{Mean Residual Life}

The probability that the time of age $t$ survives an additional interval of length $x$ is $R(x / t)=r(T>x+t / T>t)=\operatorname{Pr}(T>$ $x+t) / \operatorname{Pr}(T>t)=R(X+t) / R(t) \cdot R(x / t)$ is called the conditional survivor function of the item at age $t$. The mean residual life (MRL) of the item at age $t$ is $\operatorname{MRL}(t)=\mu(t)=$ $\int_{0}^{\infty} R(x / t) d x=(1 / R(t)) \int_{t}^{\infty} R(x) d x$.

Definition 8. Let $X$ and $Y$ be two nonnegative fuzzy random variables with continuous fuzzy distribution functions $F$ and $G$, respectively, and with hazard rate functions $r$ and $q$, respectively. Then $X$ is said to be smaller than $Y$ in the hazard rate order denoted as $\left(X \leq_{\mathrm{hr}} Y\right)$ if $r\left(x_{\alpha}^{L}\right) \leq q\left(x_{\alpha}^{L}\right)$ and $r\left(x_{\alpha}^{U}\right) \leq$ $q\left(x_{\alpha}^{U}\right)$ for each $\alpha \in(0,1]$.

Definition 9. Let $m_{F}(t)$ and $m_{G}(t)$ denote the fuzzy mean residual life of $X$ and $Y$, respectively. We define the fuzzy mean residual life ordering between $X$ and $Y$ denoted as $X \leq_{\mathrm{mrl}} Y$ if $m_{F}(t) \leq m_{G}(t) \forall t \in[0, \infty)$ and $\alpha \in(0,1]$.

Theorem 10. Let $X$ and $Y$ be two nonnegative fuzzy random variables with fuzzy hazard rate functions $\lambda_{F}(t)$ and $\lambda_{G}(t)$ and fuzzy mean residual life functions $m_{F}(t)$ and $m_{G}(t)$, respectively. If $X \leq_{h r} Y$, then $X \leq_{m r l} Y$.
Proof. By stipulation $X \leq_{\mathrm{hr}} Y$. Then, for $\alpha \in(0,1]$,

$$
\begin{aligned}
& \left(\lambda_{F}(t)\right)_{\alpha}^{L} \geq\left(\lambda_{G}(t)\right)_{\alpha}^{L}, \\
& \left(\lambda_{F}(t)\right)_{\alpha}^{U} \geq\left(\lambda_{G}(t)\right)_{\alpha}^{U}, \\
& \left(m_{F}(t)\right)_{\alpha}=\left\lfloor\left(m_{F}(t)\right)_{\alpha}^{L},\left(m_{F}(t)\right)_{\alpha}^{U}\right\rfloor \\
& =\left[\frac{\int_{t}^{\infty}(\bar{F}(u))_{\alpha}^{L} d u}{(\bar{F}(t))_{\alpha}^{L}}, \frac{\int_{t}^{\infty}(\bar{F}(u))_{\alpha}^{U} d u}{(\bar{F}(t))_{\alpha}^{U}}\right] \\
& =\left[\int_{0}^{\infty} \exp \left(-\int_{t}^{t+x}\left(\lambda_{F}(u)\right)_{\alpha}^{L} d u\right) d x,\right. \\
& \left.\int_{0}^{\infty} \exp \left(-\int_{t}^{t+x}\left(\lambda_{F}(u)\right)_{\alpha}^{U} d u\right) d x\right] \\
& \leq\left[\int_{0}^{\infty} \exp \left(-\int_{t}^{t+x}\left(\lambda_{G}(u)\right)_{\alpha}^{L} d u\right) d x,\right. \\
& \left.\int_{0}^{\infty} \exp \left(-\int_{t}^{t+x}\left(\lambda_{G}(u)\right)_{\alpha}^{U} d u\right) d x\right]=\left\lfloor\left(m_{G}(t)\right)_{\alpha}^{L},\right. \\
& \left.\left(m_{G}(t)\right)_{\alpha}^{U}\right\rfloor=\left(m_{G}(t)\right)_{\alpha}, \\
& \left(m_{F}(t)\right)_{\alpha} \leq\left(m_{G}(t)\right)_{\alpha} \\
& \Longrightarrow \bigcup_{\alpha \in(0,1]} \alpha\left(m_{F}(t)\right)_{\alpha} \leq \bigcup_{\alpha \in(0,1]} \alpha\left(m_{G}(t)\right)_{\alpha} \\
& \Longrightarrow m_{F}(t) \leq m_{G}(t) \\
& \Longrightarrow X \leq_{\mathrm{mrl}} Y
\end{aligned}
$$

for $\alpha \in(0,1]$.

This completes the proof.

\section{Bivariate Characterization of Hazard Rate Ordering of Fuzzy Random Variables}

In actuarial science, finance, and economics stochastic orderings are employed to compare the appealing nature of different risks and are essential in the theory of decision making under uncertainty. Applications are known in numerous scientific fields like queuing theory, reliability theory, demography, and Biostatistics. Stronger stochastic orders include the likelihood ratio order and the hazard rate order. These representations explicitly involve pairwise interchange of variables. These orderings have interesting applications in single machine stochastic scheduling problem, queueing network, and problems involving stochastic life times.

In this section, the focus of this study is to constitute bivariate functional representations for the hazard rate ordering which explicitly involves pairwise interchange of fuzzy random variables.

Wang and Zhang [12] have discussed that $X(\omega)$ is a fuzzy random variable if and only if $X_{\alpha}(\omega)=\left[X_{\alpha}^{L}(\omega), X_{\alpha}^{U}(\omega)\right]$ is a random interval for each $\alpha \in(0,1]$ and $X(\omega)=\bigcup_{\alpha \in(0,1]} \alpha$, 
$X_{\alpha}(\omega)=\bigcup_{\alpha \in(0,1]} \alpha\left[X_{\alpha}^{L}(\omega), X_{\alpha}^{U}(\omega)\right]$ where, for any $\alpha \in(0,1]$, $X_{\alpha}^{L}(\omega)=\inf X_{\alpha}(\omega)=\inf \{x \in R ; X(\omega)(x) \geq \alpha\}$ and $X_{\alpha}^{U}(\omega)=$ $\operatorname{Sup} X_{\alpha}(\omega)=\operatorname{Sup}\{x \in R ; X(\omega)(x) \geq \alpha\}$ with $X(\omega)(x)$ being the membership function of $X(\omega)$.

The concept of probability density function of a fuzzy random variable was proposed by Wu [13]. Let $X_{\alpha}^{L}$ and $X_{\alpha}^{U}$ have the same continuous probability density function $f(x)$. For any fuzzy observation $\widetilde{X}$ of a fuzzy random variable $X$ $(X(\omega)=\widetilde{X})$. The $\alpha$-level set $\widetilde{X}_{\alpha}=\left[\widetilde{X}_{\alpha}^{L}, \widetilde{X}_{\alpha}^{U}\right]$, where $\widetilde{X}_{\alpha}^{L}$ and $\widetilde{X}_{\alpha}^{U}$ are the observations of $\widetilde{X}_{\alpha}^{L}$ and $\widetilde{X}_{\alpha}^{U}$, respectively. $\widetilde{X}_{\alpha}^{L}(\omega)=$ $\widetilde{X}_{\alpha}^{L}$ and $\widetilde{X}_{\alpha}^{U}(\omega)=\widetilde{X}_{\alpha}^{U}$ are continuous with respect to $\alpha$ for fixed $\omega .\left[\widetilde{X}_{\alpha}^{L}, \widetilde{X}_{\alpha}^{U}\right]$ is continuously decreasing with respect to $\alpha .\left[\widetilde{X}_{\alpha}^{L}, \widetilde{X}_{\alpha}^{U}\right]$ is the disjoint union of $\left[\widetilde{X}_{\alpha}^{L}, \widetilde{X}_{1}^{U}\right]$ and $\left(\widetilde{X}_{1}^{U}, \widetilde{X}_{\alpha}^{U}\right]$ with $\widetilde{X}_{1}^{L}=\widetilde{X}_{1}^{U}$. For any real number $x \in\left[\widetilde{X}_{\alpha}^{L}, \widetilde{X}_{\alpha}^{U}\right] x=\widetilde{X}_{\beta}^{L}$ or $x=\widetilde{X}_{\beta}^{U}$ for some $\beta \geq \alpha$. Thus for any $x \in\left[\widetilde{X}_{\alpha}^{L}, \widetilde{X}_{\alpha}^{U}\right]$ we can associate a probability density function $f(x)$.

If we construct an interval

$$
\begin{gathered}
A_{\alpha}=\left\lfloor\min \left\{\min _{\alpha \leq \beta \leq 1} f\left(\tilde{x}_{\beta}^{L}\right), \min _{\alpha \leq \beta \leq 1} f\left(\tilde{x}_{\beta}^{U}\right)\right\},\right. \\
\left.\max \left\{\max _{\alpha \leq \beta \leq 1} f\left(\widetilde{x}_{\beta}^{L}\right), \max _{\alpha \leq \beta \leq 1} f\left(\widetilde{x}_{\beta}^{U}\right)\right\}\right\rfloor
\end{gathered}
$$

then this interval will contain all probability density functions associated with each of $x \in\left[\widetilde{X}_{\alpha}^{L}, \widetilde{X}_{\alpha}^{U}\right]$. The probability density function of $\widetilde{X}$ is $\widetilde{f}(\widetilde{x})$ and the membership function of $\tilde{f}(\tilde{x})$ is denoted by $\mu_{\tilde{f}(x)}(r)=\sup _{0 \leq \alpha \leq 1} \alpha 1_{A_{\alpha}}(r)$. Let $X(\omega)=$ $\bigcup_{\alpha \in(0,1]} \alpha\left[X_{\alpha}^{L}(\omega), X_{\alpha}^{U}(\omega)\right]$ be a fuzzy random variable. If the probability distribution functions of $X_{\alpha}^{L}(\omega)$ and $X_{\alpha}^{U}(\omega)$ are $f_{X_{\alpha}^{L}}(\omega)$ and $f_{X_{\alpha}^{U}}(\omega)$, then the probability density function of the fuzzy random variable $X$ is

$$
F_{X}(\cdot)=\bigcup_{\alpha \in(0,1]} \alpha\left\lfloor f_{X_{\alpha}^{L}}(\cdot), f_{X_{\alpha}^{U}}(\cdot)\right\rfloor .
$$

Definition 11. Let $X$ and $Y$ be two nonnegative fuzzy random variables with probability density functions $f_{X}(x)$ and $f_{Y}(y)$, respectively. Let $\geq_{\text {lr }}$ denote the likelihood ratio ordering. We say $X \geq_{1 \mathrm{r}} Y$ if and only if $f_{X_{\alpha}^{L}}(x) f_{y_{\alpha}^{L}}(y) \geq f_{X_{\alpha}^{L}}(y) f_{y_{\alpha}^{L}}(x)$ and $f_{X_{\alpha}^{U}}(x) f_{y_{\alpha}^{U}}(y) \geq f_{X_{\alpha}^{U}}(y) f_{y_{\alpha}^{U}}(x)$ for each $x \geq y$, and $\alpha \in(0.1]$.

Theorem 12. Let $X$ and $Y$ be fuzzy random variables and let $={ }_{d}$ denote the equality in distribution. Then $X \geq_{l r} Y$ if and only if for two independent fuzzy random variables $\widehat{X}$ and $\widehat{Y}$ such that $\widehat{X}={ }_{d} X$ and $\widehat{Y}={ }_{d} Y$ one has $\operatorname{Eg}(\widehat{X}, \widehat{Y}) \geq E g(\widehat{Y}, \widehat{X}) ; \forall g \in G_{h r}$ [10].

Lemma 13. Let $X$ and $Y$ be two random variables. We say $\geq_{h r} Y \Leftrightarrow f_{X}(y) / \bar{F}_{X}(x) \leq f_{Y}(y) / \bar{F}_{Y}(x), \forall x \geq y \Leftrightarrow \bar{F}_{X}(y) /$ $\bar{F}_{X}(x) \leq \bar{F}_{Y}(y) / \bar{F}_{Y}(x), \forall x \geq y[8]$.

Definition 14. Let $X$ and $Y$ be two nonnegative fuzzy random variables with probability density functions $f_{X}(x)$ and $f_{Y}(y)$, respectively, and their survival functions are $\bar{F}_{X}(x)$ and $\bar{F}_{Y}(y)$.
We say $X \geq_{\mathrm{hr}} Y$ if and only if

$$
\begin{aligned}
\frac{\left(f_{X}(y)\right)_{\alpha}^{L}}{\left(\bar{F}_{X}(x)\right)_{\alpha}^{L}} & \leq \frac{\left(f_{Y}(y)\right)_{\alpha}^{L}}{\left(\bar{F}_{Y}(x)\right)_{\alpha}^{L}} \\
\frac{\left(f_{X}(y)\right)_{\alpha}^{U}}{\left(\bar{F}_{X}(x)\right)_{\alpha}^{U}} & \leq \frac{\left(f_{Y}(y)\right)_{\alpha}^{U}}{\left(\bar{F}_{Y}(x)\right)_{\alpha}^{U}}
\end{aligned}
$$

for each $x \geq y$, and $\alpha \in(0,1]$.

Definition 15. Let $G_{\mathrm{hr}}$ be the collection of bivariate function $g: R^{2} \rightarrow R$ which is defined as follows:

$$
G_{\mathrm{hr}}=\{g(x, y) ; g(x, y) \geq g(y, x) ; \forall x \geq y\} .
$$

Theorem 16. Let $X$ and $Y$ be fuzzy random variables and let $={ }_{d}$ denote the equality in distribution. Then $X \geq_{h r} Y$ if and only if for two independent fuzzy random variables $\widehat{X}$ and $\widehat{Y}$ such that $\widehat{X}={ }_{d} X$ and $\widehat{Y}={ }_{d} Y$ one has $E g(\widehat{X}, \widehat{Y}) \geq E g(\widehat{Y}, \widehat{X}) ; \forall g \in$ $G_{h r}$.

Proof. Let $E g(\widehat{X}, \widehat{Y}) \geq E g(\widehat{Y}, \widehat{X}) ; \forall g \in G_{\text {hr. }}$. Let $1_{\{\{\}}$denote the indicator function.

Let $g(x, y)=1\{u \leq x \leq u+\Delta u, v-\Delta v \leq y \leq v\}$ where $u \geq v, \Delta u>0$, and $\Delta v>0$ are given constants. Obviously $g(x, y) \geq g(y, x)$ and hence $g \in G_{\mathrm{hr}}$.

By stipulation $E g(\widehat{X}, \widehat{Y}) \geq E g(\widehat{Y}, \widehat{X})$.

This shows that

$$
\begin{aligned}
& E g\left(\bigcup_{\alpha \in(0,1]} \alpha\left[\widehat{X}_{\alpha}^{L}, \widehat{X}_{\alpha}^{U}\right], \bigcup_{\alpha \in(0,1]} \alpha\left[\widehat{Y}_{\alpha}^{L}, \widehat{Y}_{\alpha}^{U}\right]\right) \\
& \quad \geq E g\left(\bigcup_{\alpha \in(0,1]} \alpha\left[\widehat{Y}_{\alpha}^{L}, \widehat{Y}_{\alpha}^{U}\right], \bigcup_{\alpha \in(0,1]} \alpha\left[\widehat{X}_{\alpha}^{L}, \widehat{X}_{\alpha}^{U}\right]\right) .
\end{aligned}
$$

This implies

$$
\begin{aligned}
& E g\left(\bigcup_{\alpha \in(0,1]} \alpha\left[\widehat{X}_{\alpha}^{L}, \widehat{Y}_{\alpha}^{L}\right], \bigcup_{\alpha \in(0,1]} \alpha\left[\widehat{X}_{\alpha}^{U}, \widehat{Y}_{\alpha}^{U}\right]\right) \\
& \quad \geq E g\left(\bigcup_{\alpha \in(0,1]} \alpha\left[\widehat{Y}_{\alpha}^{L}, \widehat{X}_{\alpha}^{L}\right], \bigcup_{\alpha \in(0,1]} \alpha\left[\widehat{Y}_{\alpha}^{U}, \widehat{X}_{\alpha}^{U}\right]\right), \\
& E g\left(\widehat{X}_{\alpha}^{L}, \widehat{Y}_{\alpha}^{L}\right) \\
& \quad=\int_{u}^{u+\Delta u} \int_{v-\Delta v}^{v}\left(\bar{F}_{X}(x)\right)_{\alpha}^{L}\left(\bar{F}_{Y}(y)\right)_{\alpha}^{L} d y d x \\
& \geq E g\left(\widehat{Y}_{\alpha}^{L}, \widehat{X}_{\alpha}^{L}\right) \\
& \quad=\int_{u}^{u+\Delta u} \int_{v-\Delta v}^{v}\left(\bar{F}_{X}(y)\right)_{\alpha}^{L}\left(\bar{F}_{Y}(x)\right)_{\alpha}^{L} d y d x .
\end{aligned}
$$

Dividing both sides by $\Delta u \Delta v$ and letting $\Delta u, \Delta v \rightarrow 0$, we obtain

$$
\left(\bar{F}_{X}(x)\right)_{\alpha}^{L}\left(\bar{F}_{Y}(y)\right)_{\alpha}^{L} \geq\left(\bar{F}_{X}(y)\right)_{\alpha}^{L}\left(\bar{F}_{Y}(x)\right)_{\alpha}^{L} ;
$$


Similarly, we can prove

$$
\begin{aligned}
&\left(\bar{F}_{X}(x)\right)_{\alpha}^{U}\left(\bar{F}_{Y}(y)\right)_{\alpha}^{U} \geq\left(\bar{F}_{X}(y)\right)_{\alpha}^{U}\left(\bar{F}_{Y}(x)\right)_{\alpha}^{U} ; \\
& \forall x \geq y .
\end{aligned}
$$

then $X \geq_{\mathrm{hr}} Y$.

To prove the converse, let $g \in G_{\mathrm{hr}}$.

$$
\text { Let } \Delta g(x, y)=g(x, y)-g(y, x) \text {. Consider }
$$

$$
\begin{aligned}
E \Delta & g(\widehat{X}, \widehat{Y})=\int_{y} \int_{x} \Delta g(x, y) f_{x}(x) f_{y}(y) d x d y \\
& =\int_{y} \int_{x \geq y} \Delta g(x, y)\left[f_{X_{\alpha}^{L}}(x) f_{y_{\alpha}^{L}}(y)\right. \\
& \left.-f_{X_{\alpha}^{L}}(y) f_{y_{\alpha}^{L}}(x)\right] d x d y \\
& =\int_{y} \int_{x \geq y} \frac{\partial}{\partial x} \Delta g(x, y)\left[\left(\bar{F}_{X}(x)\right)_{\alpha}^{L} f_{y_{\alpha}^{L}}(y)\right. \\
& \left.-f_{X_{\alpha}^{L}}(y)\left(\bar{F}_{Y}(x)\right)_{\alpha}^{L}\right] d x d y \geq 0 .
\end{aligned}
$$

See [10]. This implies $E \Delta g\left(\widehat{X}_{\alpha}^{L}, \widehat{Y}_{\alpha}^{L}\right) \geq 0$;

$$
\text { i.e., } E \Delta g\left(\bigcup_{\alpha \in(0,1]} \alpha \widehat{X}_{\alpha}^{L}, \bigcup_{\alpha \in(0,1]} \alpha \widehat{Y}_{\alpha}^{L}\right) \geq 0 \text {. }
$$

Similarly,

$$
E \Delta g\left(\bigcup_{\alpha \in(0,1]} \alpha \widehat{X}_{\alpha}^{U}, \bigcup_{\alpha \in(0,1]} \alpha \widehat{Y}_{\alpha}^{U}\right) \geq 0
$$

This shows that

$$
\begin{aligned}
& E \Delta g\left(\bigcup_{\alpha \in(0,1]} \alpha\left[\widehat{X}_{\alpha}^{L}, \widehat{X}_{\alpha}^{U}\right], \bigcup_{\alpha \in(0,1]} \alpha\left[\widehat{Y}_{\alpha}^{L}, \widehat{Y}_{\alpha}^{U}\right]\right) \geq 0, \\
& \text { i.e., } E \Delta g(\widehat{X}, \widehat{Y}) \geq 0, \\
& \text { i.e., } E(g(\widehat{X}, \widehat{Y})-g(\widehat{Y}, \widehat{X})) \geq 0, \\
& \text { i.e., } E g(\widehat{X}, \widehat{Y}) \geq E g(\widehat{Y}, \widehat{X}) ; \quad \forall g \in G_{\mathrm{hr}} .
\end{aligned}
$$

\section{Conclusion}

In this paper, the hazard rate of fuzzy random variables has been determined. The interwovenness of fuzzy hazard rate orderings and fuzzy mean residual life orderings is established. As a result, it is established that increasing failure rates in the fuzzy sense imply the decrease in fuzzy mean residual lives. It helps the consumers make decision for selecting the products. Bivariate functional representations for the hazard rate ordering of fuzzy random variables explicitly involving pairwise interchange of variables have also been obtained.

\section{Conflict of Interests}

The authors declare that there is no conflict of interests regarding the publication of this paper.

\section{References}

[1] H. Kwakernaak, "Fuzzy random variables. I. Definitions and theorems," Information Sciences, vol. 15, no. 1, pp. 1-29, 1978.

[2] M. L. Puri and D. A. Ralescu, "Fuzzy random variables," Journal of Mathematical Analysis and Applications, vol. 114, no. 2, pp. 409-422, 1986.

[3] J. M. Ruiz and J. Navarro, "Characterization of distributions by relationships between failure rate and mean residual life," IEEE Transactions on Reliability, vol. 43, no. 4, pp. 640-644, 1994.

[4] R. C. Gupta, "On the mean residual life function in survival studies," in Statistical Distribution in Scientific works, C. Tailie, G. P. Patil, and B. A. Baldessar, Eds., vol. 79 of NATO Advanced study Institutes Series, pp. 327-334, Reidel, Dordrecht, The Netherlands, 1981.

[5] R. C. Gupta and H. Olcay Akman, "Mean residual life function for certain types of non-monotonic ageing," Communications in Statistics. Stochastic Models, vol. 11, no. 1, pp. 219-225, 1995.

[6] M. S. Finkelstein, "On the shape of the mean residual lifetime function," Applied Stochastic Models in Business and Industry, vol. 18, no. 2, pp. 135-146, 2002.

[7] M. G. H. Akbari, A. H. Rezaei, and Y. Waghei, "Statistical inference about the variance of fuzzy random variables," The Indian Journal of Statistics B, vol. 71, no. 2, pp. 206-221, 2009.

[8] J. G. Shanthikumar and D. D. Yao, "Bivariate characterization of some stochastic order relations," Advances in Applied Probability, vol. 23, no. 3, pp. 642-659, 1991.

[9] T. Itoh and H. Ishii, "One machine scheduling problem with fuzzy random due-dates," Fuzzy Optimization and Decision Making, vol. 4, no. 1, pp. 71-78, 2005.

[10] J. E. L. Piriyakumar and S. Ramasubramanian, "Bivariate characterization of stochastic orderings of fuzzy random variables," in Proceedings of the International Conference in Management Sciences and Decision Making, pp. 25-31, Tamkang University, Taipei, Taiwan, 2010.

[11] M. Rausand and A. Hoyla, System Reliability Theory, Models, Statistical Methods and Applications, John Wiley \& Sons, 2004.

[12] G.-Y. Wang and Y. Zhang, "The theory of fuzzy stochastic processes," Fuzzy Sets and Systems, vol. 51, no. 2, pp. 161-178, 1992.

[13] H.-C. Wu, "Probability density functions of fuzzy random variables," Fuzzy Sets and Systems, vol. 105, no. 1, pp. 139-158, 1999. 


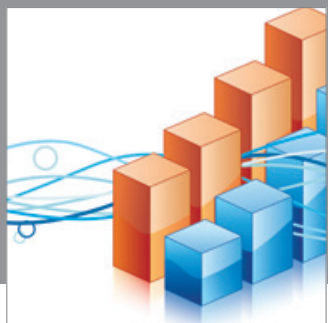

Advances in

Operations Research

mansans

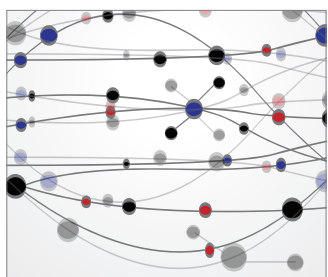

The Scientific World Journal
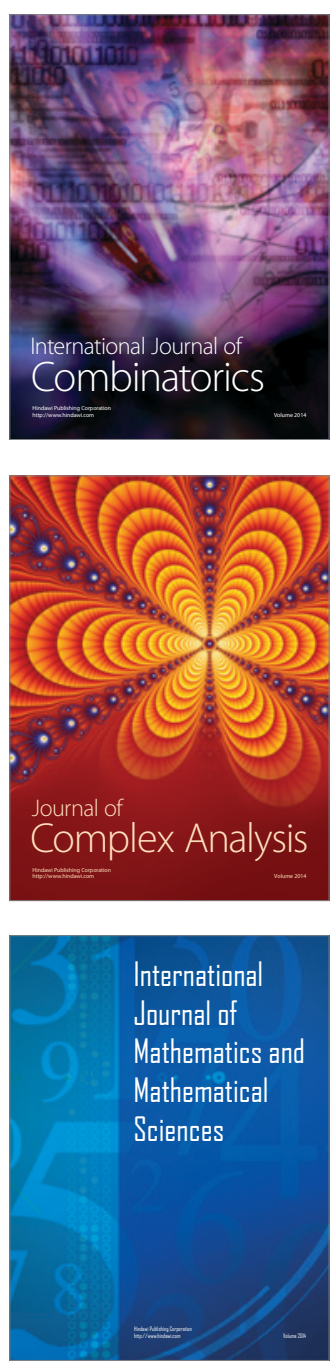
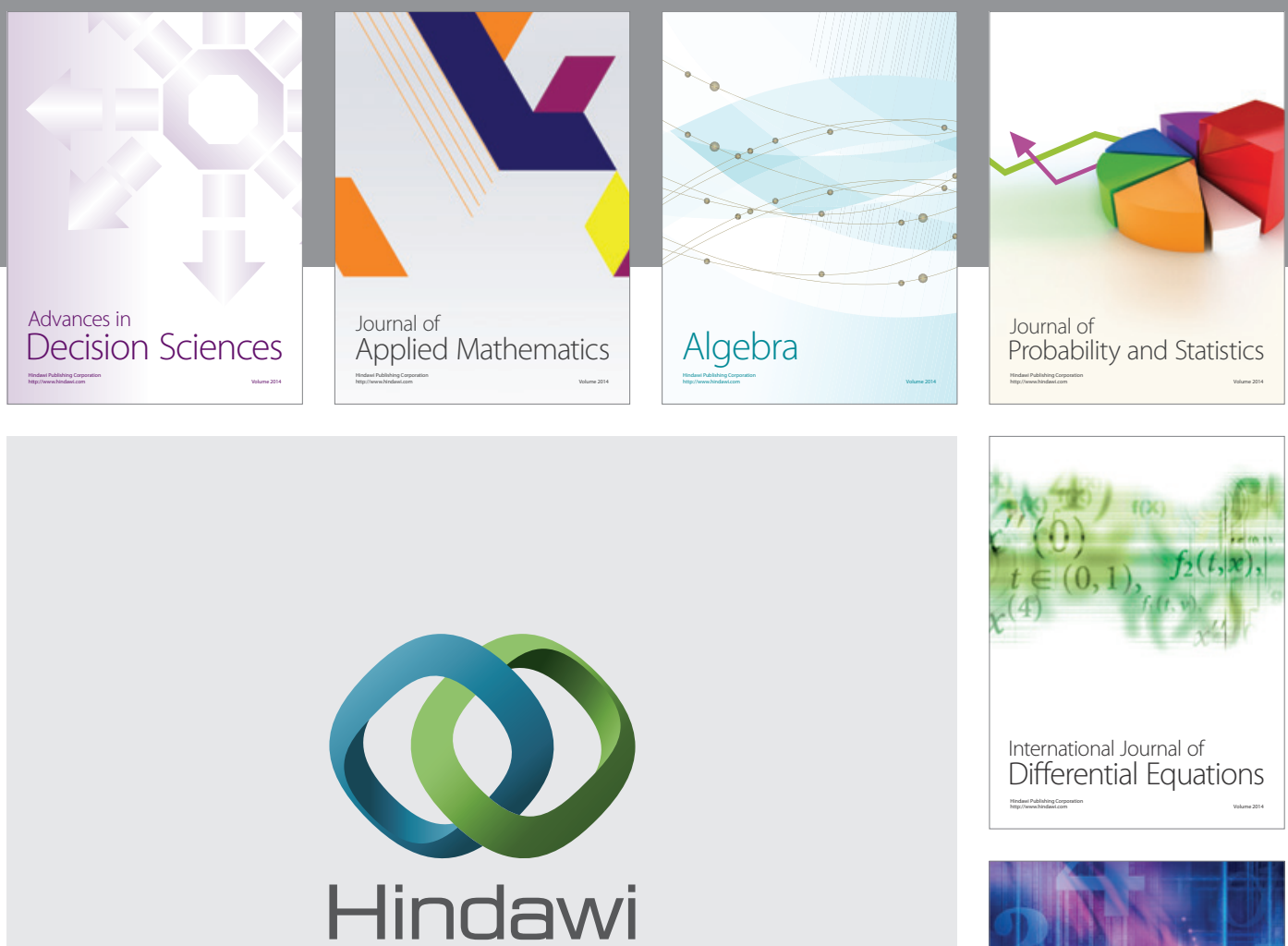

Submit your manuscripts at http://www.hindawi.com
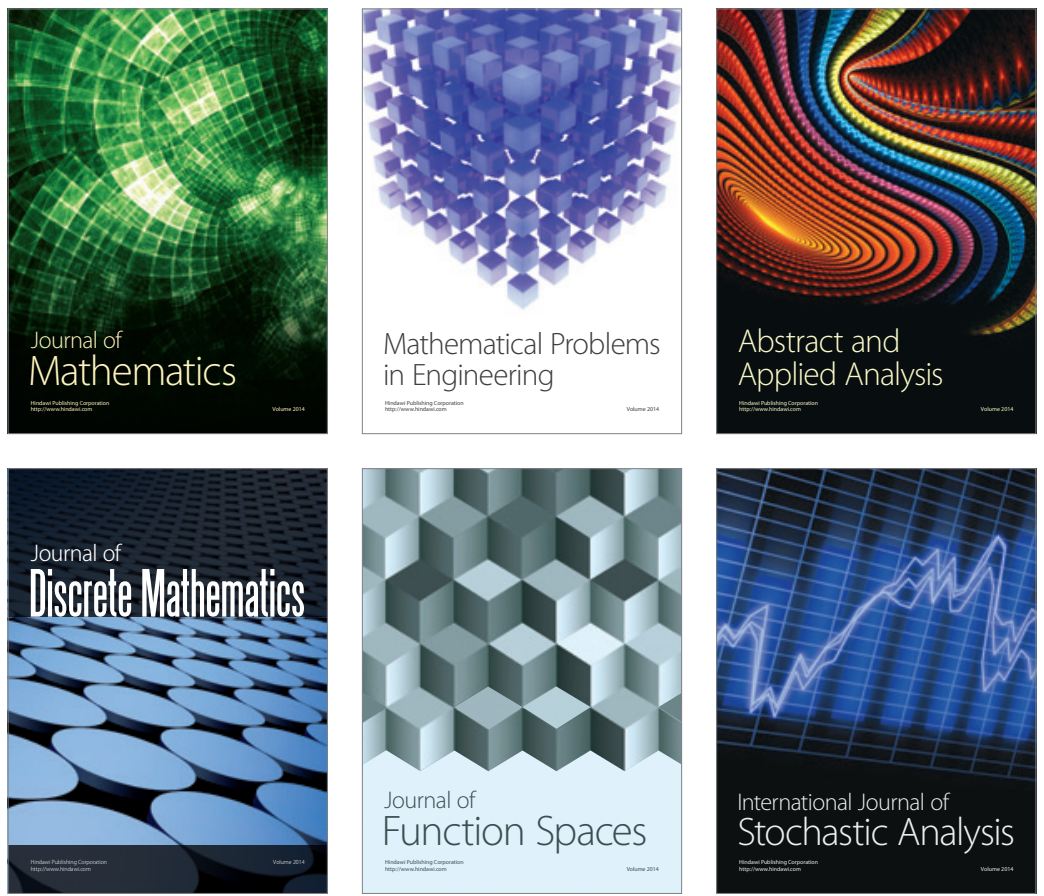

Journal of

Function Spaces

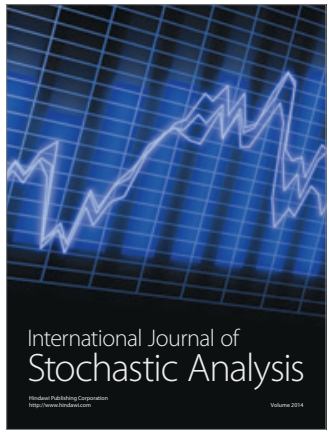

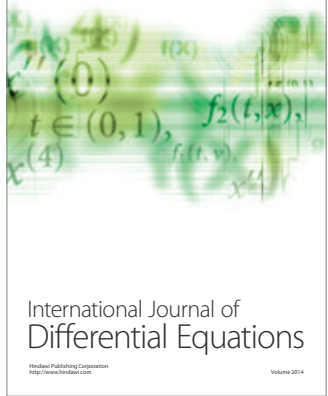
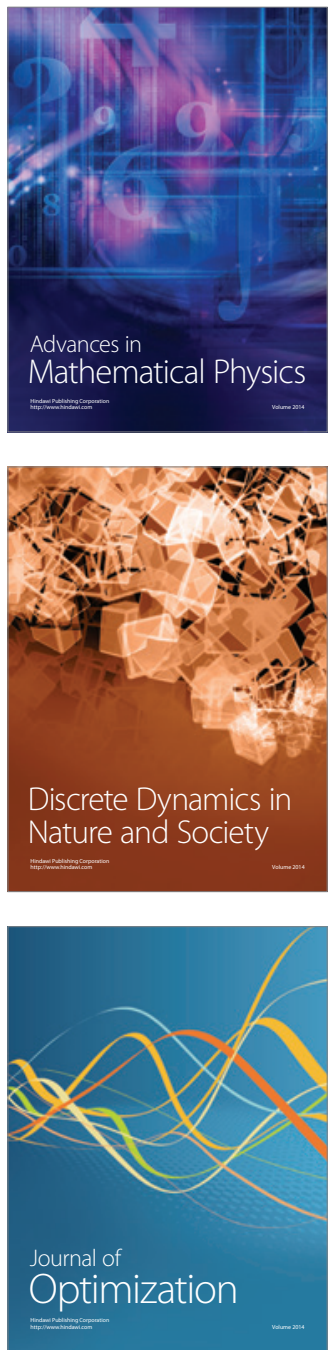